\title{
Fluid- and biomechanical analysis of ascending thoracic aorta aneurysm with concomitant aortic insufficiency
}

\author{
F. Condemi ${ }^{1,2,3}$, S. Campisi ${ }^{4}$, M. Viallon ${ }^{4,5}$, T. Troalen $^{6}$, G. Xuexin ${ }^{7}$, A.J. Barker ${ }^{8}$, M. Markl $^{8}$, \\ P. Croisille ${ }^{4,5}$, O. Trabelsi ${ }^{1,2,3}$, C. Cavinato ${ }^{1,2,3}$, A. Duprey ${ }^{4}$, S. Avril ${ }^{1,2,3}$ \\ ${ }^{1}$ Ecole Nationale Supérieure des Mines de Saint-Etienne, CIS-EMSE, SAINBIOSE, F-42023 Saint-Etienne, \\ France; ${ }^{2}$ INSERM U1059, SAINBIOSE, F-42023 Saint-Etienne, France ; ${ }^{3}$ Université de Lyon, F-69000 Lyon, \\ France ; ${ }^{4} \mathrm{CHU}$ Hôpital Nord, Saint-Etienne, France; ${ }^{5}$ Univ Lyon, UJM-Saint-Etienne, INSA, CNRS UMR 5520, \\ INSERM U1206, CREATIS, F-42023, Saint-Etienne, France; ${ }^{6}$ Siemens Healthcare, France ; ${ }^{7}$ Circle Cardiovascu- \\ lar Imaging Inc., Panarctic Plaza; ${ }^{8}$ Northwestern University, Chicago, IL, USA
}

The research presented in this paper was carried out at Univ Lyon, INSERM U1059, Mines Saint-Etienne, SAINBIOSE, F-42023, 158 cours Fauriel, 42023 SAINT-ETIENNE cedex 2, France.

Running Head: Biomechanical analysis of ascending aorta aneurysm

$\begin{array}{ll}\text { Corresponding author: } & \text { Francesca Condemi } \\ & \text { Univ Lyon, INSERM U1059, } \\ & \text { Mines Saint-Etienne, SAINBIOSE, F-42023, } \\ & 158 \text { cours Fauriel, } \\ & 42023 \text { SAINT-ETIENNE cedex 2, France } \\ & \text { Phone: }+33477426667 \\ & \text { Fax }:+33477420000 \\ & \text { Email: francesca.condemi@emse.fr }\end{array}$

Word count: 6000 words 


\section{Abstract}

We present a comprehensive and original framework for the biomechanical analysis of patients affected by ascending thoracic aorta aneurysm and aortic insufficiency. Our aim is to obtain crucial indications about the role played by deranged hemodynamics on the ATAAs risk of rupture. Computational fluid dynamics analysis was performed using patient-specific geometries and boundary conditions derived from 4D MRI. Blood flow helicity and wall shear stress descriptors were assessed. A bulge inflation test was carried out in vitro on the 4 ATAAs after surgical repair. The healthy volunteers showed no eccentric blood flow, a mean TAWSS of $1.5 \pm 0.3 \mathrm{~Pa}$ and mean OSI of $0.325 \pm 0.025$. In 3 aneurismal patients, jet flow impingement on the aortic wall resulted in large TAWSS values and low OSI which were amplified by the AI degree. However, the tissue strength did not appear to be significantly reduced. The fourth patient, which showed the lowest TAWSS due to the absence of jet flow, had the smallest strength in vitro. Interestingly this patient presented a bovine arch abnormality. Jet flow impingement with high WSS values is frequent in ATAAs and our methodology seems to be appropriate for determining whether it may increase the risk of rupture or not.

Key Terms: 4D MRI, computational fluid dynamics, wall shear stress, mechanical inflation tests and wall strength.

\section{Introduction}

Ascending thoracic aorta aneurysm (ATAA) is the $19^{\text {th }}$ common cause of human death. ${ }^{7}$ To avoid catastrophic rupture, prophylactic surgery is the recommendation, whereby the risk of mortality can be as great as $5 \%$. It is therefore the goal of the community to better risk stratify this patient population. ${ }^{27}$ In clinical practice, maximum diameter is the standard indicator to estimate risk of rupture, with a critical diameter threshold of $5.5 \mathrm{~cm} .{ }^{23}$ However, for aneurysms with a diameter smaller than $5.5 \mathrm{~cm}$, negative outcomes (rupture, dissection and death) before surgical repair do exist, with an incidence of $5-10 \%{ }^{7}$

Biomechanical studies have shown that ATAA results in disturbed aortic hemodynamics and mechanical weakening of the aortic wall. ${ }^{5,34}$ However, there is still a lack 
of insight on how disturbed hemodynamics and mechanical weakening may be related.

To date, the most studied hemodynamic parameter in the progression of vessel wall diseases is the wall shear stress (WSS) due to its key role in the gene expression and extracellular matrix remodeling. ${ }^{2,36}$ Previous studies have shown that helical and disordered blood flow determines low WSS levels in the area of the maximum ATAA dilation. ${ }^{5}$ However, little is still known about the influence of the secondary aortic regurgitation on the disturbed ATAA hemodynamics, even though the presence of $\mathrm{Al}$ is a common scenario among patients affected by ATAA. ${ }^{10}$ The combination of $4 \mathrm{D}$ flow $\mathrm{MRI}$ and computational fluid dynamics (CFD) represents a promising method for hemodynamic analysis of aortic aneurysms in the presence of $\mathrm{Al}^{6}$

In addition, a number of researchers have developed in vitro tests to identify the mechanical and rupture properties of the ATAA tissue that can be collected during surgical repair. ${ }^{12}$ Among these tests, we showed that the bulge inflation test combined with optoelectronic devices is a powerful approach to evaluate these properties with more physiological loading than simple tensile tests. ${ }^{12}$

One step further is to investigate the possible contribution of the non-homeostatic WSS, obtained from CFD analyses, on the wall mechanical and rupture properties obtained from the bulge inflation test.

The objective of this paper is to set up the methodological framework to pass this step by observing and analyzing the aortic blood flow in the particular scenario of patients affected by ATAA and Al. We first present our methodology combining 4D flow MRI and CFD models and use it to evaluate the aortic flow pattern and the aortic WSS distribution of 2 healthy volunteers and 4 patients affected by ATAA and AI. We then derive the ATAAs wall strength with the bulge inflation test.

\section{Material and Methods}

\section{Subject Enrollment and Study Design}

The study was approved by the Institutional Review Board of the University Hospital Center of St. Etienne. 
Two healthy subjects (age $=33 \pm 3$ years old) with no history of cardiovascular diseases were enrolled in the study after informed consent. In addition, four patients with a history of ATAA were enrolled, after informed consent, to measure the impact of ATAA and valve disease on the fluid- and tissue mechanical properties of these individuals. Patient 1 is a 58 year old gentleman affected by moderate stenosis, bicuspid aortic valve (BAV) and moderate-severe aortic valve regurgitation (AI II-III grade). This patient was diagnosed with ATAA and a maximum diameter $\left(D_{M A X}\right)$ of $52 \mathrm{~mm}$. Patient 2 is a 68 year old man with tricuspid aortic valve (TAV) affected by severe aortic valve regurgitation (AI III grade). The $D_{M A X}$ was equal to $50 \mathrm{~mm}$. Patient 3 is a 77 year old man with BAV affected by severe aortic valve regurgitation (AI III grade). The $D_{M A X}$ was of $49 \mathrm{~mm}$. Patient 4 is a 60 year old man with TAV affected by moderate-severe aortic valve regurgitation (AI II-III grade). The $D_{M A X}$ was equal to 48 $\mathrm{mm}$. At the 4D MRI examination a "bovine arch" variation of the aortic arch branching was found. ${ }^{20}$

The patients' demographic information and the main ATAA geometrical dimensions which are associated to the aneurismal structural configuration are summarized in Table 1. The deformation diameter rate is calculated as: ${ }^{36}$

$$
\chi=\frac{D_{M A X}}{d_{\text {proximal_neck }}}
$$

where $D_{M A X}$ is the maximum aneurysm diameter and $d_{\text {proximal_neck }}$ is the nondeformed ascending aorta (AAO) diameter.

[Placement of Table 1]

Three case studies were considered:

Study 1. The CFD analysis of two "baseline" thoracic aortas (Subject 1 and Subject 2 in Table 1) was implemented and verified using the 4D MRI data of two volunteers.

Study 2. The CFD analysis of the pathologic thoracic aortas was implemented and verified using the 4D MRI data of four patients (Patient 1, Patient 2, Patient 3 and Patient 4) affected by ATAA and Al. 
Study 3. The bulge inflation test was performed on the aneurismal tissue of the four patients recruited in this analysis who underwent ATAA surgical repair at the University Hospital Center of St. Etienne.

\section{D MRI data acquisition and post-processing}

The 2 healthy volunteers and the 4 patients were scanned on a 3T MR scanner (Siemens Magnetom Prisma) without contrast agent using a 4D flow protocol and sequence. ${ }^{37}$ The $3 \mathrm{~T}$ images acquisition was performed with a true spatial resolution of $1.9 \times 1.9 \times 2.2 \mathrm{~mm}^{3}$, field of view $(F O V)=360 \mathrm{~mm}, \mathrm{BW}=740 \mathrm{~Hz} /$ pixel, flip angle $=8^{\circ}$, $\mathrm{TE} / \mathrm{TR} / \mathrm{Tl}=2.9 / 39.2 / 150 \mathrm{~ms}$, venc $=350 \mathrm{~cm} / \mathrm{s}$ and phase duration=39,2 $\mathrm{ms}$ fixing the number of cardiac phases that hence depends on the cardiac cycle of the patient (ranging between 21-28 phases). A prospective ECG gating was used, the number of slices and rectangular FOV could be adjusted depending on the patient geometry.

The 4D flow MRI data were used to obtain the boundary conditions of the CFD model and then to verify the numerical results (Figure 1).

The 4D flow MR data analysis and visualization was performed using cvi42 $\Theta$ prototype 4DFlow module ( $\mathrm{cmr}^{42}$, Circle Cardiovascular Imaging Inc., Calgary, Canada). ${ }^{8}$

[Placement of Figure 1]

\section{Numerical Model}

\section{Geometry and mesh}

CRIMSON (CardiovasculaR Integrated Modelling and SimulatiON) software was used to build the late systolic phase 3D geometries starting from the 4D MRI images. ${ }^{11}$ The CAD models included the aortic arch, the descending aorta and the apicoaortic branches (brachiocephalic artery, BCA, left common carotid artery, LCC, and left subclavian artery, LSUB, as shown in Figure 1).

Then, the 3D geometries were imported in Ansys-Fluent (ANSYS ${ }$ ) Academic Research, Release 17.0) and were discretized using an unstructured tetrahedral mesh (Figure 1). The mesh error was calculated as the difference in percentage of the pressure drop and the TAWSS between the coarser (element size $5 \mathrm{~mm}$ ) and the finest mesh (element size $1 \mathrm{~mm}$ ). A mesh error smaller than $2 \%$ was considered ac- 
ceptable. All data presented in the paper were calculated using the grids with the highest resolution (Table 2).

[Placement of Table 2]

\section{Boundary conditions and Time dependent solution}

The pulsatile flow behaviors in the patient-specific geometries were analyzed by solving incompressible continuity and Navier-Stokes equations. Patient-specific maps of velocity profiles were extracted from the flow-MRI and then used to define the inflow boundary condition at the inlet of the aorta $\left(\mathrm{AAO}_{\text {inlet }}\right.$, Figure $\left.2 \mathrm{~A}\right)$ by using an in-house code written in Matlab (The Mathworks Inc, Natick, Mass). ${ }^{41}$

The patient-specific pulsatile flow rate obtained from 4D MRI analysis was set as the outlet boundary condition at the apico-aortic branches (BCA, LCC and LSUB, Figure 2B). ${ }^{31}$ A multi-scale approach was implemented to describe the hemodynamics at the descending aorta outlet by coupling the 3D domain with a reduced order model. The three-elements Windkessel model is a zero-dimensional electric circuit analogue which uses two resistors to represent the vasculature resistance and a capacitor to represent the vessel compliance (Figure 2A). ${ }^{11}$ Given a target diastolic and systolic pressure, the value of the impedance $\left(Z_{C}\right)$, the distal resistance $(R)$ and the capacitor (C) were tuned to produce the same flow rate waveform obtained from the 4D MRI data analyses. ${ }^{40}$

Aortic walls were assumed to be rigid and impermeable, and a no-slip condition, $\mathrm{v}_{\text {wall }}=0$, was used. Blood was treated as a non-Newtonian fluid using Carreau model. $^{16}$

The transient numerical model was solved by the finite volume method using the PISO algorithm solver and a time step of $5 \mathrm{~ms}$. The convergence of the solution was assessed for residuals error equal to $10^{-4}$. Three cardiac cycles were run to ensure fully developed flow and to eliminate the unsteady effect of the initial transient. ${ }^{40}$

[Placement of Figure 2] 


\section{Quantitative analysis of hemodynamics indices}

The velocity maps and the velocity profiles in the dilated region of the aorta obtained from the CFD results and the 4D MRI data were analyzed. Two planes of interest were defined $\left(A T A A_{\text {middle }}\right.$ in the region of the maximum dilatation and $A T A A_{\text {end }}$ in the region downstream the aneurysm, Figure $2 A$ ) by taking into account the diameter at these planes and the distance from the aorta inlet. The flow eccentricity (Flow eccentricity) was defined by the Euclidean distance between the vessel centerline and the center velocity of the forward flow normalized to the lumen diameter. The center velocity was calculated as the average position of the pixel in the lumen vessel weighted by the velocity value. ${ }^{35}$ Flow $_{\text {eccentricity }}$ equal to 0 indicates that flow is centrally distributed with respect to the vessel centerline. Flow eccentricity equal to 1 indicates that the flow is eccentric and impinges against the vessel walls. The Floweccentricity was calculated at the end of the blood flow deceleration (time $c$ along the $A A o_{\text {inlet }}$ flow rate waveform in Figure $2 \mathrm{C}$ ) which is the time frame when the aortic valve closes under physiologic conditions.

The helical structures of the aortic blood flow along the cardiac cycle were measured by calculating the Localized Normalized Helicity $(\mathrm{LNH})$ as: ${ }^{14,15,28,30}$

$$
\operatorname{LNH}(s ; t)=\frac{V(s ; t) \cdot \omega(s ; t)}{|V(s ; t)||\omega(s ; t)|}
$$

where $\mathrm{V}$ is the velocity vector, $\omega$ is the vorticity vector, $\mathrm{s}$ is the position and $\mathrm{t}$ is the time. The LNH variates between -1 and 1 (left-handed and right-handed rotation, respectively), describing the local value of the cosine of the angle between the velocity and the vorticity vectors. Purely helical flow results in high values of $\mathrm{LNH}( \pm 1)$ and symmetric flow occurs when $\mathrm{LNH}$ is equal to zero. ${ }^{15,28,30}$

The index $h_{2}$ was calculated to define the total amount of helicity in the aortic volume. The index $h_{3}$ which ranges between -1 and +1 was calculated to define the orientation of the helical structures in the domain $(-1$ indicates only left-handed rotation, 1 indicates only right-handed rotation and 0 indicates reflectional symmetry). ${ }^{14,29}$

\section{Wall shear stress analysis}

The wall shear stress (WSS), the time-averaged wall shear stress (TAWSS) and the Oscillatory Shear Index (OSI) were first calculated along the entire domain of the aor- 
tic wall in the different case studies. Then, the analysis of the results was focused on the value and the distribution of the WSS descriptors along the ATAA wall.

The TAWSS magnitude was measured along three cardiac cycles by integrating each nodal WSS magnitude over the cardiac cycle as: ${ }^{13}$

$$
T A W S S=\frac{1}{T} \int_{0}^{T} W S S d t
$$

where $T$ is the period of the cardiac cycle and WSS is the instantaneous wall shear stress.

The OSI was used to identify the presence of high oscillatory WSS direction during the cardiac cycle and was calculated as: $:^{24}$

$$
O S I=0.5\left[1-\left(\frac{\left|\int_{0}^{T} W S S(s, t) \cdot d t\right|}{\int_{0}^{T}|W S S(s, t)| \cdot d t}\right)\right]
$$

\section{Bulge inflation tests}

A bulge inflation test was performed on the 4 ATAAs after surgical repair. Briefly, a $50 \mathrm{~mm}$ aortic sample was cut in the axial direction from the greater curvature of each ATAA specimen. Then, a flat square piece consisting of the tunica intima, media and adventitia was clamped in a bulge inflation device after carefully removing the loose connective tissue. ${ }^{12}$ The aortic tissue specimen was inflated by injecting water at a constant rate of $15 \mathrm{ml} / \mathrm{min}$ until the tissue ruptured (Figure 1). Simultaneously, the pressure was measured using a digital manometer (WIKA, DG-10) and the process was recorded using a SDIC system to reconstruct the 3D shape of the inflated tissues. The collected images were analyzed using ARAMIS (GOM, v. 6.2.0). ${ }^{38}$ The 3D shape of the inflated ATAA layer captured in vitro, was numerically discretized in order to create a mesh across the 3D shape. The Cauchy stress $(\sigma)$ at each element of the mesh was derived assuming that the tested samples behave as a pure membrane. ${ }^{12}$ The ultimate strength is measured by the maximum stress that the wall can withstand while being loaded. The stress at the stage in which the rupture occurred (trup) was derived as: ${ }^{12}$ 


$$
\sigma_{\text {rup }}=\sigma_{1}\left(t_{\text {rup }}\right) \cos ^{2} \theta+\sigma_{2}\left(t_{\text {rup }}\right) \sin ^{2} \theta
$$

where $\sigma_{1}$ and $\sigma_{2}$ are, respectively, the circumferential and axial components of the Cauchy stress tensor at the center of the specimen; and $\theta$ is the angle between the vector normal to the crack line $\left(e_{\theta}\right)$ and the axial direction of the aorta $\left(e_{2}\right.$, as shown in Figure 1). The lower is the $\sigma_{\text {rup }}$, the larger is the risk of rupture.

\section{Results}

\section{CFD Analysis Verification}

Flow $_{\text {eccentricity }}$ at the end of the systolic deceleration (time $c$ along the $A A o_{\text {inlet }}$ flow rate waveform in Figure $2 \mathrm{C}$ ) calculated from the CFD studies was verified against the results obtained from the 4D MRI analysis (Table 3).

[Placement of Table 3]

\section{Blood flow eccentricity}

Figure 3 shows the 3D streamlines distribution obtained, respectively, from the 4D MRI acquisitions and the CFD studies during the deceleration phase. The two volunteers (Subject 1 and Subject 2) showed laminar flow patterns distribution with no eccentric blood flow. The 3D streamlines were relatively symmetric and evenly distributed with respect to the vessel centerline indicating undisturbed flow (Figure $3 \mathrm{~A}$ and Figure 3B). For the two BAV patients (Patient 1 and Patient 3), Floweccentricity was found in the region of the bulge and downstream the region of the aneurysm, during the deceleration phase (Table 3). Floweccentricity caused the displacement of the highest velocity flow away from the aortic centerline and blood flow impingement against the aneurysmal wall (Figure 3C and Figure 3D). For the TAV patients (Patient 2 and Patient 4), the highest Floweccentricity was found in the region downstream the maximum dilatation (Table 3). Blood flow impingement was found only for Patient 2 downstream the region of the bulge (Figure 3E and Figure 3F).

[Placement of Figure 3] 


\section{Helicity}

Figure 4 shows the $\mathrm{LNH}$ isosurfaces averaged over the cardiac cycle (left-handed helical structure in blue, right-handed in red). $\mathrm{LNH}$ (with a threshold $\mathrm{LNH}= \pm 0.3$ ) was used to visualize the presence of helical structures in the bulk flow.

For Subject 1, helical structures were observed in the bulk flow (Figure 4A). The $h_{2}$ value was equal to 3.73. The value of $h_{3}$ was equal to -0.04 (Table 4). For Subject 2 helical structures were found along the aorta arch (Figure 4B). The $h_{2}$ value was equal to 4.12. The value of $h_{3}$ was equal to 0.03 .

For Patient 1, who had a BAV phenotype, helical counter-rotating structures were found upstream the aorta inlet and in the region of the bulge (Figure 4C). The $h_{2}$ index was more than three times the value found in the two physiological subjects (Subject 1 and Subject 2) and was equal to 10.34. The $h_{3}$ index was equal to -0.16 (Table 4). For Patient 3, who had a BAV phenotype, helical flow occurred in the posterior area of the proximal $A A o$ and in both the anterolateral region of the aortic arch (Figure 4D). The $h_{2}$ index was two times the physiological values and was equal to 7.21 , while $h_{3}$ was equal to -0.12 (Table 4 ).

For Patient 2, who had a TAV phenotype, helical counter-rotating structures were found in the right and left anterolateral region of the aortic arch (Figure 4E). The $\mathrm{h}_{2}$ index was equal to 3.58 , while $h_{3}$ was equal to -0.05 (Table 4). For Patient 4 , who had a TAV phenotype, LNH showed helical structures distributed along the aortic arch walls (Figure 4F). The $h_{2}$ index was equal to 4.31 , while $h_{3}$ was equal to -0.05 (Table 4).

[Placement of Table 4]

[Placement of Figure 4]

\section{WSS-indicators}

Figure 5 shows the TAWSS and the OSI distribution for all the case studies.

For Subject 1, the highest WSS in the AAo was $10 \mathrm{~Pa}$, at systole. At the deceleration time, the highest WSS in the AAo was $4 \mathrm{~Pa}$. The maximum TAWSS was $1.69 \mathrm{~Pa}$ and 
it was extended along the aortic arch (Figure 5A). The OSI value along the aortic arch was of 0.3 (Figure $5 \mathrm{~A}$ ).

For Subject 2, the systolic highest WSS in AAo was $12 \mathrm{~Pa}$. At the deceleration time, the highest WSS in AAo was $7 \mathrm{~Pa}$. The maximum TAWSS was $0.99 \mathrm{~Pa}$ and it was distributed from the proximal region to the distal region of the aortic arch. The OSI value along the aortic arch was of 0.35 (Figure 5B).

The highest values for the WSS-indicators were found for the two BAV patients (Patient 1 and Patient 3). For Patient 1, the systolic maximum WSS was found in the left side of the aortic arch and was equal to $21.30 \mathrm{~Pa}$. During the deceleration the highest WSS was of $8.96 \mathrm{~Pa}$. During diastole, the WSS was found to be equal to $4.00 \mathrm{~Pa}$. In the same region was found the highest TAWSS with a peak of $5.40 \mathrm{~Pa}$ and the lowest OSI which was equal to 0.15 (Figure $5 \mathrm{C}$ ). For Patient 3 , the maximum WSS was found during the systolic phase in the bulge region and was of $12 \mathrm{~Pa}$. In the same area during the deceleration the highest WSS was of $10 \mathrm{~Pa}$. The maximum TAWSS was of $5 \mathrm{~Pa}$ and the lowest OSI was equal to 0.21 (Figure 5D).

For Patient 2, who had a TAV phenotype, the maximum systolic WSS was found in the region of the bulge and was equal to $18.20 \mathrm{~Pa}$. During the deceleration, the highest WSS was found in the region downstream the ATAA and was of $14.20 \mathrm{~Pa}$. The maximum TAWSS was 3.76 $\mathrm{Pa}$ and the lowest OSI was equal to 0.22 (Figure 5E). For Patient 4, who had a TAV phenotype, the maximum WSS was found during systole in the left side aortic arch and was of $9 \mathrm{~Pa}$. During the deceleration the highest WSS was of $4 \mathrm{~Pa}$. The maximum TAWSS was $3 \mathrm{~Pa}$ and the lowest OSI was equal to 0.25 (Figure 4F).

[Placement of Figure 5]

\section{Wall mechanical properties}

The results showed that an increase of the ATAA asymmetry contributed to an increase of TAWSS. Among the two BAV patients (patient 1 and patient 3), a trend toward an increase in wall strength was found to be associated to a decrease of the helicity, a decrease of the TAWSS and an increase of the OSI in the region of the bulge (Table 5). The highest ultimate strength $\left(\sigma_{\text {rup }}=2.46 \mathrm{MPa}\right)$ was observed for the 
smallest ATAA radius $(49 \mathrm{~mm})$ and the lowest TAWSS $(5 \mathrm{~Pa})$ which occurred in patient 3. Among the two TAV patients (patient 2 and patient 4) the lowest strength value $\left(\sigma_{\text {rup }}=0.6 \mathrm{MPa}\right)$ was found in patient 4 who had the ATAA with the smallest diameter (diameter $=48 \mathrm{~mm}$ ). The TAWSS in the weakest region was found to be abnormally low and equal to $0.8 \mathrm{~Pa}$.

[Placement of Table 5]

\section{Discussion}

In this study, we investigate for the first time the possible correlation between the deranged hemodynamics, obtained from CFD analyses, and the ATAA mechanical and rupture properties obtained from the bulge inflation test. We first discuss what are the main determinants of TAWSS and OSI in ATAA patients and then how they may be associated with the mechanical properties of the aortic wall in ATAAs.

\section{About TAWSS and OSI}

Deranged ATAA hemodynamics is frequently associated to alterations of the arterial morphology. In this context, the aortic valve morphology is believed to play a key role in the development and in the growth of clinically significant AAo dilatations. BAV lesions are reported to change considerably the distribution and magnitude of the WSS along the ATAA. ${ }^{2,3}$ Moreover, recent studies have shown that even in the absence of ATAA, aortic annulus and root dimensions are significantly larger in patients with BAV insufficiency than patients with BAV stenosis. ${ }^{1}$

To the best of our knowledge, this is the first study which examines the hemodynamics in ATAA with concomitant Al by combining 4D MRI analyses and CFD studies. The objectives of this research were to observe the impact of secondary flow on the ATAA hemodynamics and to understand how the degree of Al can be indicative of possible rupture risk. Highest Floweccentricity and helicity were observed in patients affected by BAV and Al (Patient 1 and Patient 3). Floweccentricity induced flow impingement against the aneurysmal wall generating a non-homeostatic distribution of the WSS which was found to be higher and more extended than TAV patients. ${ }^{18,33,39}$ In these areas was found the highest TAWSS and the lowest OSI. Floweccentricity was lower in TAV patients affected by Al (Patient 2 and Patient 4) and helicity was comparable to the values found in the physiologic subjects (Subject 1 and Subject 2). In 
Patient 2 the jet flow impingement against the aortic wall was found in the region around the bulge, downstream the area of maximum dilatation. In the same region the maximum TAWSS and the minimum OSI were found. Only patient 4 had no region of jet flow impingement but the bulge for this patient developed on the inner curvature side. It can be observed that the existence of jet flow impingement and the TAWSS magnitude at its location are mostly associated with the inclination at the inlet. This inclination is quantified as the neck angle between the plane of the inlet cross section and the transverse plane of the thorax (angle $\theta$ in Figure 2A). An angle of about 25 degrees exists for healthy subjects and for patient 4 . The neck angle goes to 70 degrees for patient 1 and patient 2 who have high TAWSS. The origin of this neck angle change comes from the change of aorta morphology during the ATAA development. ${ }^{3,19}$ Patients 1, 2 and 3 have a larger curvature which may result from a larger increase of the aorta length on the outer curvature side than on the inner curvature side. Different structural properties on the inner and outer curvature sides have already been reported and possibly associated with the contact between the pulmonary artery and the aorta on the inner curvature side..$^{9}$ It should be noted that the ATAA development is associated with a decrease of the aorta axial stretch in mice models of the Marfan syndrome, ${ }^{4}$ which results from elastin fragmentation and faster collagen turnover. These effects (elastin loss, axial stretch decrease) are expected to be more pronounced in the areas exposed to larger hemodynamic loading which would be more favorable to fatigue damage of elastin and aorta remodeling. ${ }^{17,18,32}$ Moreover, this remodeling (i.e. aorta diameter increase, arch length increase and aortic unfolding) leads to functional aortic alterations such as decreased aortic distensibility and increased aortic arch pulse wave velocity. ${ }^{12,34}$

The case of patient 4 is peculiar as a smaller aneurysm diameter was observed, with growth on the inner curvature side and no significant change of curvature or of inclination angle at the inlet occurred. This may explain the lower TAWSS. However, the 4D MRI examination showed a variant of aortic "bovine arch" in which the LCC artery originates from the $\mathrm{BCA},{ }^{26}$ which is not the case of the three other cases. The "bovine arch" was found to be significantly common in patients with thoracic aortic disease and was associated with high ATAA growth rate and earlier repair. ${ }^{20}$ This more complex clinical scenario makes this patient's results very interesting to show but not comparable with the results of the other 3 patients. 
We showed that the WSS-indicators are significantly affected by the inclination angle at the inlet and by the presence of BAV associated to Al.

\section{About the association between strength and TAWSS}

Our results do not show any correlation between strength and diameter which is in agreement with our previous study on a cohort of 31 patients. $^{12}$

It also seems that no simple relationship exists between the wall strength and WSS. Large TAWSS values in the bulging region of aneurysms may even be associated with relatively large strength, as for patient 1 for instance. In addition, large strength difference between TAV and BAV patients (from 0.78 MPa in patient 2 to $2.46 \mathrm{MPa}$ in patient 3 ) is associated to relatively small TAWSS variation (from $3.76 \mathrm{~Pa}$ in patient 2 to $5.40 \mathrm{~Pa}$ in patient 3). Actually, if we showed that the wall remodeling and its subsequent changes in the aortic morphology have an important impact on the WSS, the reciprocal is less clear as is still unclear if WSS mediated mechanics affects the remodeling in the ascending thoracic aorta. ${ }^{22}$ Generally, maintenance of arterial caliber in response to increased blood pressure (to restore WSS toward normal) tends to involve vessel-level changes in vasoactivity, which is greater in muscular arteries than elastic arteries. ${ }^{21}$ There is yet little information on possible basal tone in human aorta. $^{22}$

\section{Limitations/future work}

The first limitation of this analysis is the small number of patients included. However, this 4D MRI/CFD study was intended as proof of concept for disturbed hemodynamics in ATAA region during the blood flow deceleration due to the concomitance of $\mathrm{Al}$ in different clinical scenarios including valve phenotypes and AAo irregular morphology.

The second limitation of the study is the rigid wall assumption. Actually, we focused our analysis on the TAWSS instead of the WSS at the systolic peak. This TAWSS which is calculated by integrating the instantaneous WSS over the time eliminates any temporal effect. ${ }^{25}$ Therefore, the difference in TAWSS between wall rigid and wall motion models are negligible. ${ }^{25}$ 
Moreover, we did not analyze the existence of any possible relationships between turbulent quantities (e.g., turbulent kinetic energy) and strength. We plan to achieve further analysis related to turbulence effects in a future study.

Finally, the aortic valve was not included in the geometrical model and the motion of aortic-valve leaflets over the cardiac cycle was not simulated. However, patientspecific maps of velocity profiles were extracted from 4D-MRI and then used to define the inflow boundary condition at the inlet of the aorta, as described in the "Material and Methods" section.

\section{Conclusion}

We combined 4D MRI and CFD models to evaluate the flow patterns and the WSS distribution in patients affected by ATAA and Al. Then, we measured the wall strength of the ATAAs in vitro with the bulge inflation test. Our results showed that jet flow impingement with high WSS values was common in ATAAs but the trends did not show a direct link between the presence of these high TAWSS values and the decrease of tissue strength. Future investigations will have to verify these results on a larger cohort of patients to finally elucidate the link between deranged hemodynamics and wall mechanical properties in ATAAs.

\section{Acknowledgments}

This research was supported by the European Research Council (ERC grant biolochanics, grant number 647067).

We thank Dr Morbiducci and Dr Gallo from Polytechnic of Turin (Italy) who provided insight and expertise that greatly assisted this research. We are also grateful to Ansys, Inc. for providing Ansys-Fluent (ANSYS ${ }^{\circledR}$ Academic Research, Release 17.0). 


\section{Figure Legends}

Figure 1. The figure shows a workflow overview of the analysis presented in the paper. Firstly, the 3D geometry was constructed using patient-specific images; secondly, the CFD model was built and solved using patient-specific boundary conditions from 4D MRI acquisition; thirdly, the CFD model was verified using 4D MRI data. Finally, the Cauchy stress at the stage in which the rupture occurred ( $\left.\sigma_{\text {rup }}\right)$ was derived from the bulge inflation test.

Figure 2. (A) Patient-specific velocity profile which was extracted from the flow-MRI and was used as aorta inlet boundary condition ( $\left.A A O_{\text {inlet }}\right)$. $A T A A_{\text {middle }}$ is the region of the maximum dilatation and $A T A A_{\text {end }}$ is the region downstream the aneurysm. The black arrow on the plane ATAA $A_{\text {middle }}$ represents the deviation of the blood flow with respect to the aorta centerline. The Windkessel model was used as outlet boundary condition at the descending aorta. Angle $\theta$ is the neck angle between the plane of the inlet cross section and the transverse plane of the thorax. (B) The patient-specific pulsatile flow rate obtained from $4 \mathrm{D} \mathrm{MRI}$ analysis was set as the outlet boundary condition at the apico-aortic branches (BCA, LCC and LSUB). (C) The AAoinlet flow rate waveform resulted from the velocity profile interpolation along the cardiac cycle: (a) acceleration time, (b) peak of systole, (c) end of systolic deceleration and (d) diastole.

Figure 3. Spatial overview of the blood flow velocity during the blood flow deceleration (time $c$ in Figure 2C) derived from the 4D MRI post-processing data (at the top of the Figure) and from the CFD models (at the bottom of the Figure). (A) Subject 1 data, (B) Subject 2 data, (C) Patient 1 data, (D) Patient 3 data, (E) Patient 2 data, (F)

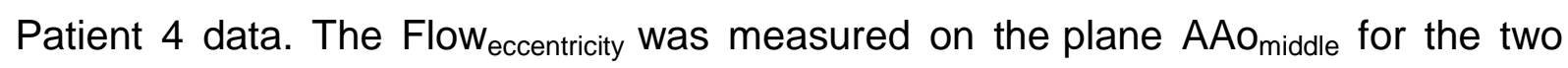
subjects and on the planes ATAA $_{\text {middle }}$ and ATAA $_{\text {end }}$ for all the patients.

Figure 4. The Figure shows the LNH isosurfaces averaged over the cardiac cycle (left-handed helical structure in blue, right-handed in red). LNH (with a threshold $\mathrm{LNH}= \pm 0.3$ ) was used to visualize the presence of helical structures in the bulk flow. (A) Subject 1 data, (B) Subject 2 data, (C) Patient 1 data, (D) Patient 3 data, (E) Patient 2 data, (F) Patient 4 data. 
Figure 5. TAWSS (at the top of the Figure) and OSI (at bottom of the Figure) resulting from the CFD analysis for all the case studies. (A) Subject 1 data, (B) Subject 2 data, (C) Patient 1 data, (D) Patient 3 data, (E) Patient 2 data, (F) Patient 4 data. 


\section{References}

1. Al-Atassi, T., M. Hynes, B. Sohmer, B.-K. Lam, T. Mesana, and M. Boodhwani. Aortic root geometry in bicuspid aortic insufficiency versus stenosis: implications for valve repair. Eur. J. Cardiothorac. Surg. 47:e151e154, 2015.

2. Barker, A.J., C. Lanning, and R. Shandas. Quantification of hemodynamic wall shear stress in patients with bicuspid aortic valve using phase-contrast MRI. Ann. Biomed. Eng. 38:788-800, 2010.

3. Barker, A.J., M. Markl, J. Bürk, R. Lorenz, J. Bock, S. Bauer, J. SchulzMenger, and F. von Knobelsdorff-Brenkenhoff. Bicuspid aortic valve is associated with altered wall shear stress in the ascending aorta. Circ. Cardiovasc. Imaging. 5:457-466, 2012.

4. Bellini, C., A. Korneva, L. Zilberberg, F. Ramirez, D. B. Rifkin, and J. D. Humphrey. Differential ascending and descending aortic mechanics parallel aneurysmal propensity in a mouse model of Marfan syndrome. J. Biomech. 49:2383-2389, 2016.

5. Bürk, J., P. Blanke, Z. Stankovic, A.J. Barker, M. Russe, J. Geiger, A. Frydrychowicz, M. Langer, and M. Markl. Evaluation of 3D blood flow patterns and wall shear stress in the normal and dilated thoracic aorta using flowsensitive 4D CMR. J. Cardiovasc. Magn. Reson. 14:84-84, 2012.

6. Callaghan, F.M., J. Karkouri, K. Broadhouse, M. Evin, D. F. Fletcher, and S. M. Grieve. Thoracic aortic aneurysm: 4D flow MRI and computational fluid dynamics model. Comput. Methods Biomech. Biomed. Engin. 18:1894-1895, 2015. 
7. Chau, K.H., and J.A. Elefteriades. Natural history of thoracic aortic aneurysms: size matters, plus moving beyond size. Prog. Cardiovasc. Dis. 56:74-80, 2013.

8. Childs, H., L. Ma, M. Ma, J. Clarke, M. Cocker, J. Green, O. Strohm, and M. G. Friedrich. Comparison of long and short axis quantification of left ventricular volume parameters by cardiovascular magnetic resonance, with ex-vivo validation. J. Cardiovasc. Magn. Reson. 13:40, 2011.

9. Choudhury, N., O. Bouchot, L. Rouleau, D. Tremblay, R. Cartier, J. Butany, R. Mongrain, and R. L. Leask. Local mechanical and structural properties of healthy and diseased human ascending aorta tissue. Cardiovasc. Pathol. 18:83-91, 2009.

10. David, T.E., C.M. Feindel, and J. Bos. Repair of the aortic valve in patients with aortic insufficiency and aortic root aneurysm. J. Thorac. Cardiovasc. Surg. 109:345-352, 1995.

11. Dillon-Murphy, D., A. Noorani, D. Nordsletten, and C.A.Figueroa. Multimodality image-based computational analysis of haemodynamics in aortic dissection. Biomech. Model. Mechanobiol. 15:857-876, 2016.

12. Duprey, A., O. Trabelsi, M. Vola, J.-P. Favre, and S. Avril. Biaxial rupture properties of ascending thoracic aortic aneurysms. Acta Biomater. 42:273285, 2016.

13. Fytanidis, D.K., J.V. Soulis, and G.D Giannoglou. Patient-specific arterial system flow oscillation. Hippokratia 18:162-165, 2014.

14. Gallo, D., D.A. Steinman, P.B. Bijari, U. Morbiducci. Helical flow in carotid bifurcation as surrogate marker of exposure to disturbed shear. J Biomech. 45(14):2398-404, 2012. 
15. Garcia, J., A. J. Barker, J. D. Collins, J. C. Carr, M. Markl. Volumetric quantification of absolute local normalized helicity in patients with bicuspid aortic valve and aortic dilatation. Magn. Res. Med. 78:689-701, 2017.

16. Gijsen, F.J.H., F.N. van de Vosse, and J.D. Janssen. The influence of the nonNewtonian properties of blood on the flow in large arteries: steady flow in a carotid bifurcation model. J. Biomech. 32:601-608, 1999.

17. Girdauskas, E., and M. Rouman. Is there any difference in aortic wall quality between patients with bicuspid aortic valve stenosis and those with bicuspid aortic valve insufficiency? Eur. J. Cardiothorac Surg. 2: 337-337, 2014.

18. Guzzardi, D.G., A.J. Barker, P. van Ooij, S.C. Malaisrie, J.J. Puthumana, D.D. Belke, H.E.M. Mewhort, D.A. Svystonyuk, S. Kang, S. Verma, J. Collins, J. Carr, R.O. Bonow, M. Markl, J.D. Thomas, P.M. McCarthy, and P.W.M. Fedak. Valve-Related Hemodynamics Mediate Human Bicuspid Aortopathy: Insights From Wall Shear Stress Mapping. J. Am. Coll. Cardiol. 66:892-900, 2015.

19. Hope, M.D., M. Sigovan, S.J. Wrenn, D. Saloner, and P. Dyverfeldt. Magnetic resonance imaging hemodynamic markers of progressive bicuspid aortic valve related aortic disease. J. Magn. Reson. Imaging 40:140-145, 2014.

20. Hornick, M., R. Moomiaie, H. Mojibian, B. Ziganshin, Z. Almuwaqqat, E. S. Lee, J. A. Rizzo, M. Tranquilli, and J. A. Elefteriades. 'Bovine' aortic arch - a marker for thoracic aortic disease. Cardiology 123:116-124, 2012.

21. Humphrey, J.D. Cardiovascular Solid Mechanics. New York, NY:Springer, 2002.

22. Humphrey, J.D., M.A. Schwartz, G. Tellides, and D.M. Milewicz. Role of mechanotransduction in vascular biology: focus on thoracic aortic aneurysms and dissections. Circ. Res. 116:1448-1461, 2015. 
23. Isselbacher, E.M. Thoracic and abdominal aortic aneurysms. Circulation 111: 816-828, 2005.

24. Ku D.N., D. P. Giddens, C.K. Zarins, S. Glagov. Pulsatile flow and atherosclerosis in the human carotid bifurcation. Positive correlation between plaque location and low oscillating shear stress. Arteriosclerosis. 5:293-302, 1985.

25. Lantz, J., J. Renner, and M. Karlsson. Wall shear stress in a subject specific human aorta - influence of fluid-structure interaction. Int. J. Appl. Mech. 03:759-778, 2011.

26. Layton, K.F., D.F. Kallmes, H.J. Cloft, E.P. Lindell, and V.S Cox. Bovine aortic arch variant in humans: clarification of a common misnomer. Am. J. Neuroradiol. 27: 1541-1542, 2006.

27. Michelena, H.I., S.K. Prakash, A. Della Corte, M.M. Bissell, N. Anavekar, P. Mathieu, Y. Bossé, G. Limongelli, E. Bossone, D.W. Benson, P. Lancellotti, E.M. Isselbacher, M. Enriquez-Sarano, T.M. 3rd Sundt, P. Pibarot, A. Evangelista, D.M. Milewicz, S.C. Body, and BAVCon Investigators. Bicuspid Aortic Valve: Identifying Knowledge Gaps and Rising to the Challenge From the International Bicuspid Aortic Valve Consortium (BAVCon). Circulation 129:2691-2704, 2014.

28. Morbiducci, U., R. Ponzini, M. Grigioni, and A. Redaelli. Helical flow as fluid dynamic signature for atherogenesis risk in aortocoronary bypass. A numeric study. J. Biomech. 40:519-534, 2007.

29. Morbiducci, U., R. Ponzini, G. Rizzo, M. Cadioli, A. Esposito, F. De Cobelli, A. Del Maschio, F.M. Montevecchi, A. Redaelli. In Vivo Quantification of Helical 
Blood Flow in Human Aorta by Time-Resolved Three-Dimensional Cine Phase Contrast Magnetic Resonance Imaging. Ann Biomed Eng. 37(3):516-31, 2009.

30. Morbiducci, U., R. Ponzini, G. Rizzo, M. Cadioli, A. Esposito, F.M. Montevecchi, A. Redaelli. Mechanistic insight into the physiological relevance of helical blood flow in the human aorta: an in vivo study. Biomech. Model. Mechanobiol. 10:339-355, 2011.

31. Morbiducci, U., R. Ponzini, D. Gallo, C. Bignardi, and G. Rizzo. Inflow boundary conditions for image-based computational hemodynamics: impact of idealized versus measured velocity profiles in the human aorta. J. Biomech. 46:102-109, 2013.

32. O'Rourke, M.F., and J. Hashimoto. Mechanical factors in arterial aging: a clinical perspective. J. Am. Coll. Cardiol. 50:1-13, 2007.

33. Pasta, S., A. Rinaudo, A. Luca, M. Pilato, C. Scardulla, T.G. Gleason, and D.A. Vorp. Difference in hemodynamic and wall stress of ascending thoracic aortic aneurysms with bicuspid and tricuspid aortic valve. J. Biomech. 46:1729-1738, 2013.

34. Redheuil, A., W.C. Yu, E. Mousseaux, A.A. Harouni, N. Kachenoura, C.O. Wu, D. Bluemke, and J.A.C Lima. Age-Related Changes in Aortic Arch Geometry: : Relationship with Proximal Aortic Function and Left Ventricular Mass and Remodeling. J. Am. Coll. Cardiol. 58: 1262-1270, 2011.

35. Sigovan, M., M.D. Hope, P. Dyverfeldt, D. Saloner. Comparison of fourdimensional flow parameters for quantification of flow eccentricity in the ascending aorta. J Magn Reson Imaging. 34(5):1226-30, 2011. 
36. Soudah, E., E. Y. K. Ng, T. H. Loong, M. Bordone, U. Pua, and S. Narayanan. CFD modelling of abdominal aortic aneurysm on hemodynamic loads using a realistic geometry with CT. Comput. Math. Methods Med. 9, 2013.

37. Strecker, C., A. Harloff, W. Wallis, and M. Markl. Flow-sensitive 4D MRI of the thoracic aorta: comparison of image quality, quantitative flow, and wall parameters at 1.5 T and 3 T. J. Magn. Reson. Imaging 36:1097-1103, 2012.

38. Trabelsi, O., F.M. Davis, J.F. Rodriguez-Matas, A.Duprey, and S. Avril. Patient specific stress and rupture analysis of ascending thoracic aneurysms. J. Biomech. 48:1836-1843, 2015.

39. Tse, K.M., P. Chiu, H.P. Lee, and P. Ho. Investigation of hemodynamics in the development of dissecting aneurysm within patient-specific dissecting aneurismal aortas using computational fluid dynamics (CFD) simulations. J. Biomech. 44:827-836, 2011.

40. Xiao, N., J.D Humphrey, C.A. Figueroa. Multi-scale computational model of three- dimensional hemodynamics within a deformable full-body arterial network. J. Comput. Phys. 244:2-40, 2013.

41. Youssefi, P., A. Gomez, Taigang H., L. Anderson, N. Bunce, R. Sharma, C.A. Figueroa, M. Jahangiri. Patient-specific computational fluid dynamics and assessment of aortic hemodynamics in a spectrum of aortic valve pathologies. J Thorac Cardiov Surg. 153(1):8-20.e3, 2017. 


\section{Figure 1}

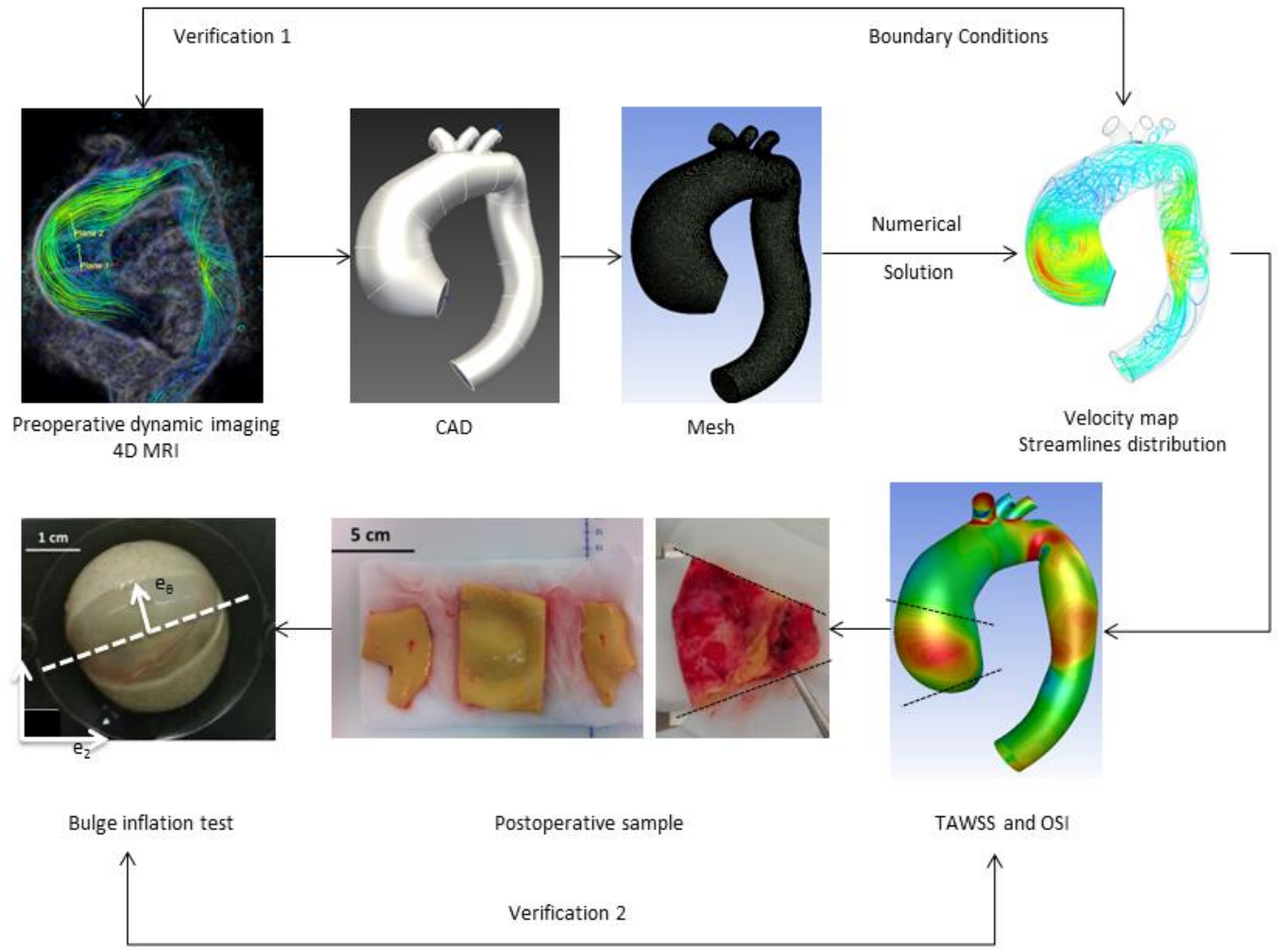




\section{Figure 2}

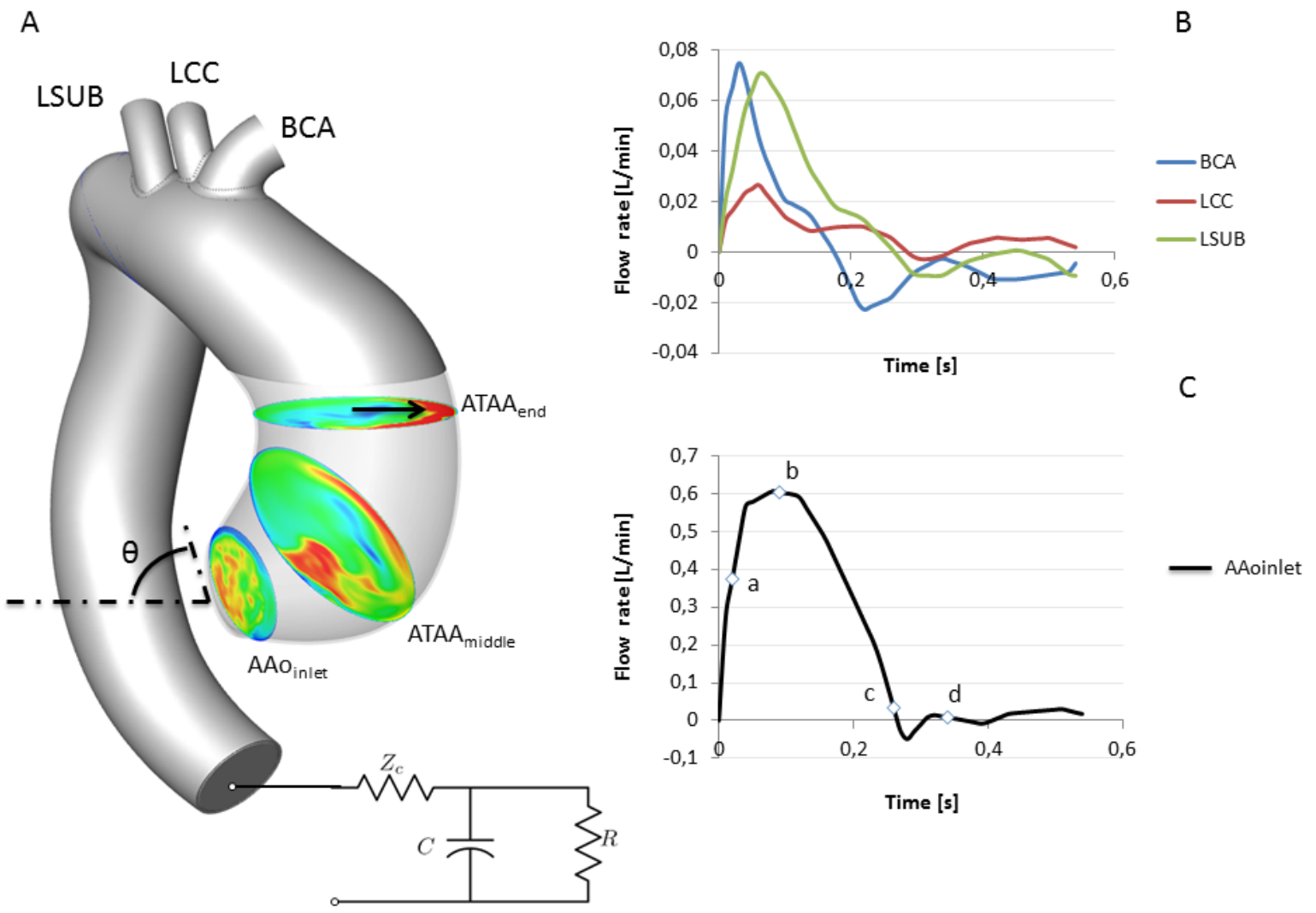


Figure 3
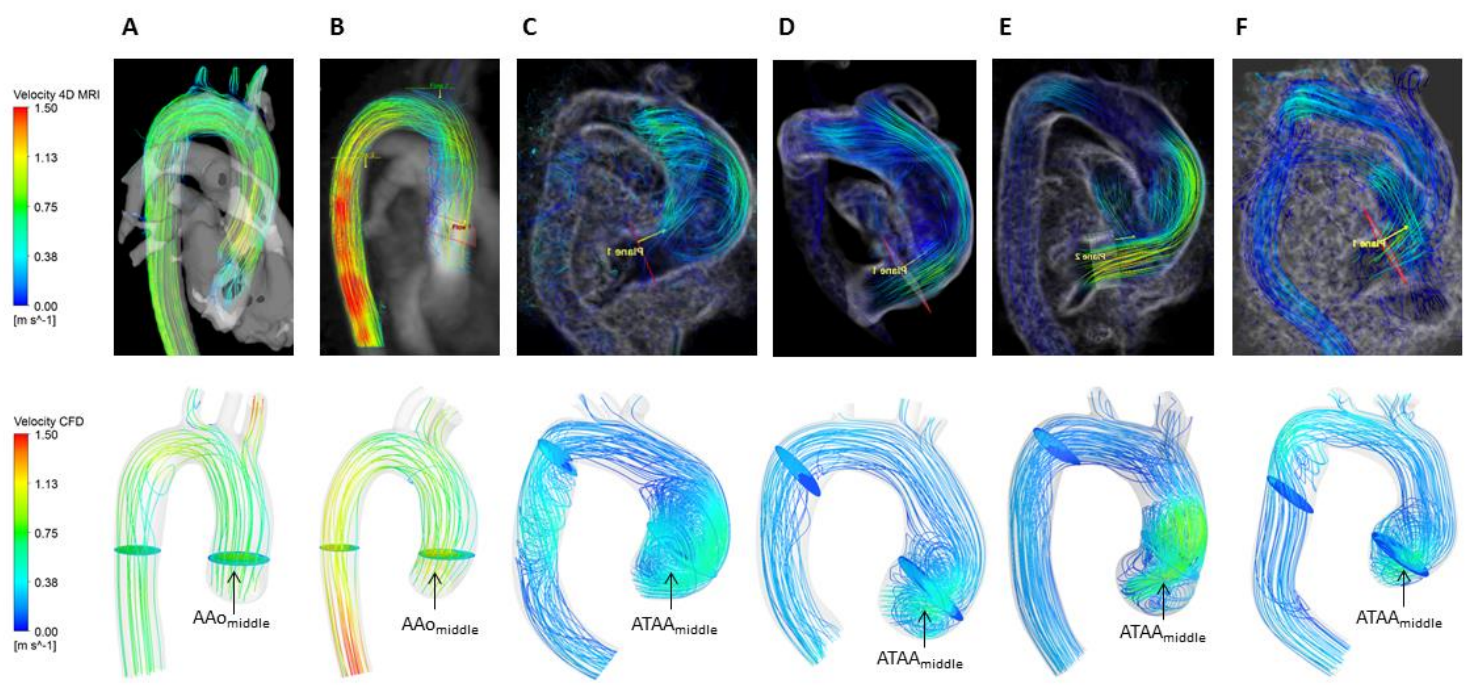

Figure 4

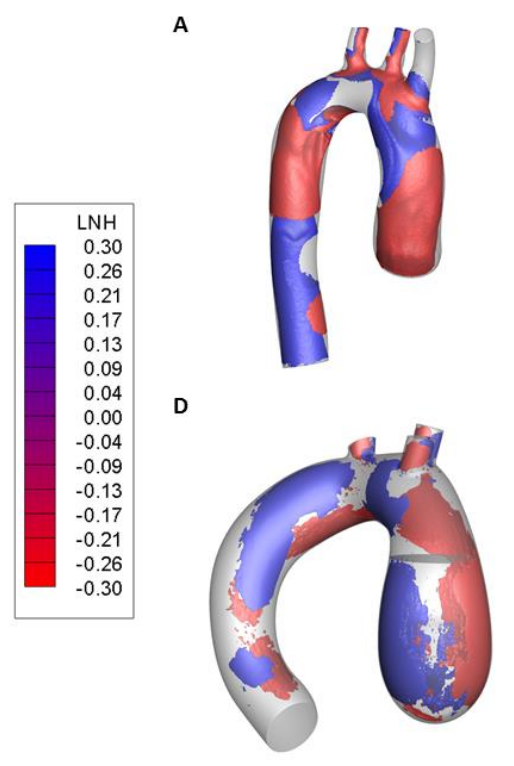

B

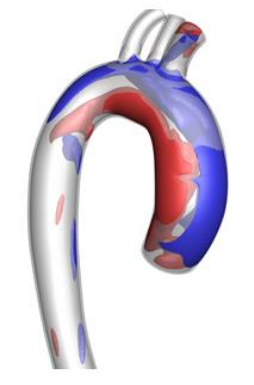

E

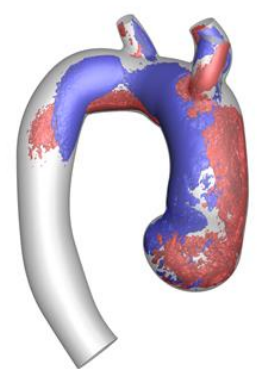

c

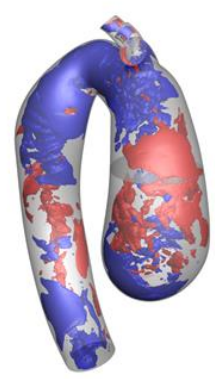

F

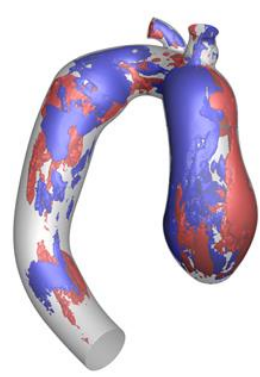


Figure 5

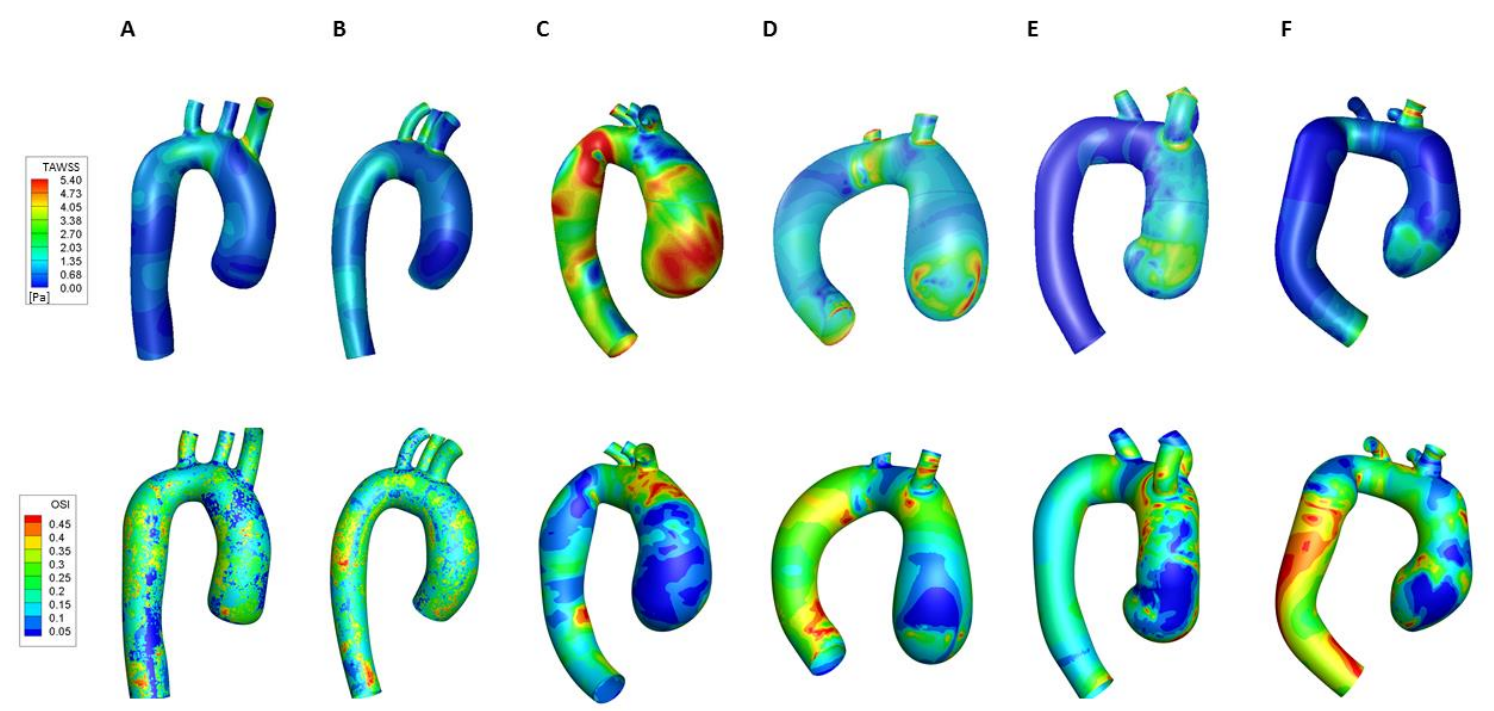


Table 1. Demographic information and ATAA geometrical dimensions.

\begin{tabular}{|c|c|c|c|c|c|c|c|c|}
\hline & & Sex/Age/Valve & $\begin{array}{c}\text { Al } \\
\text { Grade }\end{array}$ & Stenosis & $\begin{array}{r}L_{A A T A} \\
{[\mathrm{~mm}]}\end{array}$ & $\begin{array}{c}D_{M A X} \\
{[\mathrm{~mm}]}\end{array}$ & $\begin{array}{c}\boldsymbol{d}_{\text {proximal_neck }} \\
{[\mathrm{mm}]}\end{array}$ & $\chi$ \\
\hline \multirow{2}{*}{ Study 1} & $\begin{array}{l}\text { CASE 1 } \\
\text { (Subject 1) }\end{array}$ & M/36/TAV & - & - & - & 26 & 26 & 1 \\
\hline & $\begin{array}{c}\text { CASE } 2 \\
\text { (Subject 2) }\end{array}$ & F/30/TAV & - & - & - & 24 & 24 & 1 \\
\hline \multirow{4}{*}{ Study 2} & $\begin{array}{c}\text { CASE } 3 \\
\text { (Patient 1) }\end{array}$ & M/58/BAV & $\begin{array}{l}\text { II-III } \\
\text { (moderate- } \\
\text { severe) }\end{array}$ & moderate & 51 & 52 & 40 & 1.3 \\
\hline & $\begin{array}{c}\text { CASE } 4 \\
\text { (Patient 2) }\end{array}$ & M/68/TAV & $\begin{array}{c}\text { III } \\
\text { (severe) }\end{array}$ & - & 40 & 50 & 45 & 1.1 \\
\hline & $\begin{array}{c}\text { CASE } 5 \\
\text { (Patient 3) }\end{array}$ & M/77/BAV & $\begin{array}{c}\text { III } \\
\text { (severe) }\end{array}$ & - & 31 & 49 & 37 & 1.3 \\
\hline & $\begin{array}{c}\text { CASE } 6 \\
\text { (Patient 4) }\end{array}$ & M/60/TAV & $\begin{array}{c}\text { II-III } \\
\text { (moderate- } \\
\text { severe) }\end{array}$ & - & 38 & 48 & 43 & 1.1 \\
\hline
\end{tabular}

$L_{A A A}$ is the length of the artery section affected by aneurysm; $D_{M A X}$ is the maximum diameter of the aneurysm sac; $d_{\text {proximal_neck }}$ is the undeformed diameter of the ascending aorta; $\chi$ is the diameter deformation rate. For the "baseline" aortas $D_{\text {MAX }}$ is equal to $d_{\text {proximal_neck }}$.

$\mathrm{TAV}=$ tricuspid aortic valve; $\mathrm{BAV}=$ bicuspid aortic valve. 
Table 2. Mesh resolution and Windkessel parameters for the different cases

\begin{tabular}{|c|c|c|c|c|c|}
\hline & \multicolumn{2}{|c|}{ Mesh resolution } & \multicolumn{3}{c|}{ Windkessel Parameters } \\
\cline { 2 - 6 } & Elements & Nodes & $\begin{array}{c}\mathrm{Z}_{\mathrm{c}} \\
{\left[\mathrm{kg} \cdot \mathrm{m}^{-4} \cdot \mathrm{s}^{-1}\right]}\end{array}$ & $\begin{array}{c}\mathrm{C} \\
{\left[\mathrm{kg}{ }^{-1} \cdot \mathrm{m}^{4} \cdot \mathrm{s}^{2}\right]}\end{array}$ & $\begin{array}{c}\mathrm{R} \\
{\left[\mathrm{kg} \cdot \mathrm{m}^{-4} \cdot \mathrm{s}^{-1}\right]}\end{array}$ \\
\hline Subject 1 & $1.3 \mathrm{M}$ & $300 \mathrm{~K}$ & $1.36 \mathrm{e}^{+7}$ & $1.5 \mathrm{e}^{-8}$ & $2.28 \mathrm{e}^{+8}$ \\
\hline Subject 2 & $1.05 \mathrm{M}$ & $286 \mathrm{~K}$ & $1.04 \mathrm{e}^{+7}$ & $1.47 \mathrm{e}^{-8}$ & $1.77 \mathrm{e}^{+8}$ \\
\hline Patient 1 & $6.1 \mathrm{M}$ & $1.3 \mathrm{M}$ & $1.50 \mathrm{e}^{+7}$ & $1.05 \mathrm{e}^{-8}$ & $2.50 \mathrm{e}^{+8}$ \\
\hline Patient 2 & $4.3 \mathrm{M}$ & $942 \mathrm{~K}$ & $1.65 \mathrm{e}^{+7}$ & $1.58 \mathrm{e}^{-8}$ & $2.77 \mathrm{e}^{+8}$ \\
\hline Patient 3 & $3.3 \mathrm{M}$ & $716 \mathrm{~K}$ & $4.50 \mathrm{e}^{+7}$ & $2.10 \mathrm{e}^{-8}$ & $1.50 \mathrm{e}^{+8}$ \\
\hline Patient 4 & $3.0 \mathrm{M}$ & $693 \mathrm{~K}$ & $2.47 \mathrm{e}^{+7}$ & $1.05 \mathrm{e}^{-8}$ & $3.63 \mathrm{e}^{+8}$ \\
\hline
\end{tabular}

$Z_{c}$ is the impedance, $R$ is the distal resistance and $C$ is the capacitor of the Windkessel model. 


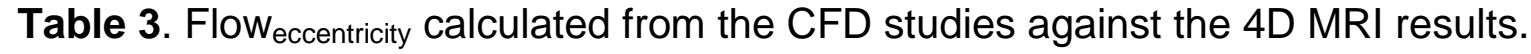

\begin{tabular}{|c|c|c|c|c|}
\hline & \multicolumn{2}{|c|}{ AAo Flow eccentricity } & & \\
\hline & 4D MRI & CFD & & \\
\hline Subject 1 & 0.018 & 0.026 & - & - \\
\hline \multirow[t]{3}{*}{ Subject 2} & $\approx 0$ & 0.002 & - & - \\
\hline & \multicolumn{2}{|c|}{ ATAA $_{\text {middle }}$ Flow $_{\text {eccentricity }}$} & \multicolumn{2}{|c|}{ ATAA $_{\text {end }}$ Flow $_{\text {eccentricity }}$} \\
\hline & 4D MRI & CFD & 4D MRI & CFD \\
\hline Patient 1 & 1.000 & 0.880 & 1.000 & 0.920 \\
\hline Patient 2 & 0.500 & 0.550 & 0.700 & 0.760 \\
\hline Patient 3 & 0.290 & 0.320 & 0.650 & 0.570 \\
\hline Patient 4 & 0.390 & 0.330 & 0.51 & 0.470 \\
\hline
\end{tabular}

ATAA $_{\text {middle }}$ is the region of the maximum dilatation; ATAA $_{\text {end }}$ is the region downstream the aneurysm. Considering as reference the inlet of the aorta, $A A o_{\text {middle }}$ is the approximately central region along the ascending thoracic aorta for Subject 1 and Subject 2. 
Table 4 Helicity, OSI and TAWSS results for the different case-studies.

\begin{tabular}{|c|c|c|c|c|}
\hline & \multicolumn{2}{|c|}{ Helicity } & \multirow{2}{*}{$O S I_{\min }$} & \multirow{2}{*}{$\begin{array}{c}\text { TAWSS }_{\max } \\
{[\mathrm{Pa}]}\end{array}$} \\
\hline & $\mathrm{h}_{2}$ & $h_{3}$ & & \\
\hline Subject 1 & 3.73 & -0.04 & 0.30 & 1.69 \\
\hline Subject 2 & 4.12 & 0.03 & 0.35 & 0.99 \\
\hline Patient 1 & 10.34 & -0.16 & 0.15 & 5.40 \\
\hline Patient 2 & 3.58 & -0.05 & 0.22 & 3.76 \\
\hline Patient 3 & 7.21 & -0.12 & 0.21 & 5.00 \\
\hline Patient 4 & 4.31 & -0.05 & 0.25 & 3.00 \\
\hline
\end{tabular}


Table 5. The ultimate strength obtained from the bulge inflation test and the TAWSS obtained from the CFD analysis across a region of $20 \mathrm{~mm}$ radius around the center of the ATAA. The total helicity, $h_{2}$.

\begin{tabular}{|c|c|c|c|c|c|c|}
\hline & $\begin{array}{c}\text { Thickness } \\
{[\mathrm{mm}]}\end{array}$ & $\begin{array}{c}\text { Diameter } \\
{[\mathrm{mm}]}\end{array}$ & $\begin{array}{c}\text { ATAA/Aortic } \\
\text { Valve } \\
\text { Morphology }\end{array}$ & $\begin{array}{c}\boldsymbol{\sigma}_{\text {rupture }} \\
\text { [MPa] }\end{array}$ & $\begin{array}{c}\text { TAWSS in the } \\
\text { center of the } \\
\text { ATAA [Pa] }\end{array}$ & $\mathbf{h}_{2}$ \\
\hline Patient 1 & 2.74 & 52 & $\begin{array}{c}\text { Asymmet- } \\
\text { ric/BAV }\end{array}$ & 1.11 & 5.40 & 10.34 \\
\hline Patient 2 & 2.20 & 50 & $\begin{array}{c}\text { Symmet- } \\
\text { ric/TAV }\end{array}$ & 0.78 & 3.76 & 3.58 \\
\hline Patient 3 & 2.14 & 49 & $\begin{array}{c}\text { Asymmet- } \\
\text { ric/BAV }\end{array}$ & 2.46 & 5.00 & 7.21 \\
\hline \multicolumn{7}{|c|}{ Bovine arch patient } \\
\hline Patient 4
\end{tabular}


Appendix A.
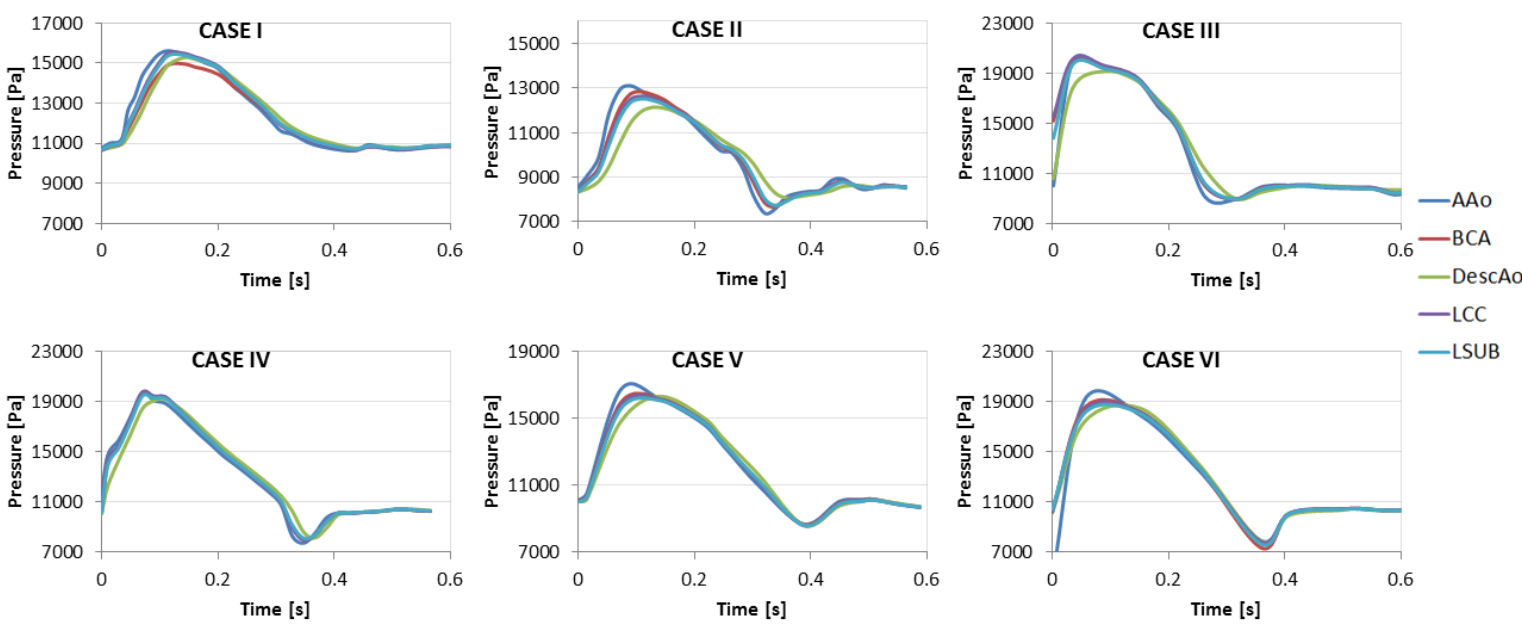

Pressure plots for the different case studies. 